\title{
Potential of Radio Telescopes as High-Frequency Gravitational Wave Detectors
}

\author{
Valerie Domcke $\odot^{1,2,3, *}$ and Camilo Garcia-Cely $\circledast^{1, \dagger}$ \\ ${ }^{1}$ Deutsches Elektronen-Synchrotron DESY, Notkestrasse 85, 22607 Hamburg, Germany \\ ${ }^{2}$ Theoretical Physics Department, CERN, 1 Esplanade des Particules, CH-1211 Geneva 23, Switzerland \\ ${ }^{3}$ Institute of Physics, Laboratory for Particle Physics and Cosmology (LPPC), \\ École Polytechnique Fédérale de Lausanne (EPFL), CH-1015 Lausanne, Switzerland
}

(Received 11 June 2020; revised 6 August 2020; accepted 7 December 2020; published 14 January 2021)

\begin{abstract}
In the presence of magnetic fields, gravitational waves are converted into photons and vice versa. We demonstrate that this conversion leads to a distortion of the cosmic microwave background (CMB), which can serve as a detector for $\mathrm{MHz}$ to $\mathrm{GHz}$ gravitational wave sources active before reionization. The measurements of the radio telescope EDGES can be cast as a bound on the gravitational wave amplitude, $h_{c}<10^{-21}\left(10^{-12}\right)$ at $78 \mathrm{MHz}$, for the strongest (weakest) cosmic magnetic fields allowed by current astrophysical and cosmological constraints. Similarly, the results of ARCADE 2 imply $h_{c}<10^{-24}\left(10^{-14}\right)$ at 3-30 GHz. For the strongest magnetic fields, these constraints exceed current laboratory constraints by about 7 orders of magnitude. Future advances in $21 \mathrm{~cm}$ astronomy may conceivably push these bounds below the sensitivity of cosmological constraints on the total energy density of gravitational waves.
\end{abstract}

DOI: 10.1103/PhysRevLett.126.021104

Gravitational waves (GWs) produced in the early Universe [1,2] can traverse cosmic distances without experiencing any interactions, making them a unique probe of very high energy physics. Since the comoving Hubble horizon grows with time, GWs produced at energies around the scale of grand unification have frequencies in the $\mathrm{MHz}$ and $\mathrm{GHz}$ regime today, far beyond the reach of the laser interferometers LIGO, VIRGO, or KAGRA. See Refs. [3-8] for some existing laboratory bounds at these frequencies.

Here we focus on searching for high-frequency GWs exploiting the (inverse) Gertsenshtein effect $[9,10]$, which describes the conversion of GWs into photons in the presence of a magnetic field (see, e.g., Refs. [7,8,11-16]). As an immediate consequence of general relativity and classical electromagnetism, this is a purely SM process. Involving gravity, the conversion probability is extremely small which may, however, be compensated by considering a "detector" of cosmological size. In fact, magnetic fields with cosmological correlation lengths might well permeate our Universe with certain astrophysical observations strongly suggesting a lower limit of order $10^{-16} \mathrm{G}$ [17-19], and the CMB setting an upper bound in the pG-nG range [20-22]. See Ref. [23] for a comprehensive review.

The pioneering study [24] proposed the inverse Gertsenshtein effect in cosmic magnetic fields to search

Published by the American Physical Society under the terms of the Creative Commons Attribution 4.0 International license. Further distribution of this work must maintain attribution to the author(s) and the published article's title, journal citation, and DOI. for GWs but neglected the plasma mass of photons, as pointed out in Ref. [25]. The idea was revisited in Ref. [26] suggesting an observable effect, however as noted in Ref. [15] decoherence effects were not correctly accounted for. More recently, Ref. [27] studied the production of GWs from CMB photons. In this Letter, we focus on CMB distortions arising from the Gertsenshtein effect during the dark ages, i.e., the period between recombination and reionization. Because of the small fraction of free electrons in this period, the effective plasma mass of the photons is suppressed, increasing the conversion probability between GWs and photons. Taking into account inhomogeneities in the thermal plasma and in the cosmic magnetic fields, we demonstrate that existing measurements of the RayleighJeans tail of the CMB spectrum, performed, e.g., by ARCADE 2 [28] and by EDGES [29], can be translated into constraints on GWs in the $\mathrm{MHz}-\mathrm{GHz}$ regime. These are competitive with, or even exceed, current laboratory constraints, depending on the assumptions on the cosmic magnetic fields.

The Gertsenshtein effect.-Calculating the conversion rate for this oscillation process requires solving Maxwell's equations for the vector potential $A^{\mu}$, describing the electromagnetic radiation, together with the linearized Einstein's equations for the metric $g_{\mu \nu}=\eta_{\mu \nu}+h_{\mu \nu}$, in which $h_{\mu \nu}$ describes the GWs. In this work we will adopt $\eta_{\mu \nu}=\operatorname{diag}(+---)$ and work with natural HeavisideLorentz units $(\hbar=c=1)$, except in this section, where we keep fundamental constants explicitly to emphasize that the Gertsenshtein effect is a classical phenomenon.

Let us ignore the Universe expansion first and consider a GW propagating in the $\hat{\mathbf{e}}_{3}$ direction inside a fixed box of 


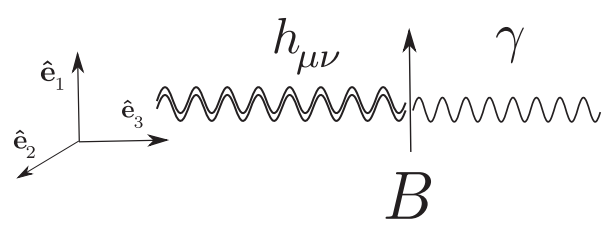

FIG. 1. The Gertsenshtein effect.

size $\Delta \ell$ that contains a uniform transverse magnetic field $B$ and a non-negligible uniform density of free electrons, $n_{e}$. Without loss of generality, we assume that the magnetic field points in the $\hat{\mathbf{e}}_{1}$ direction. See Fig. 1 . In this coordinate system we introduce $h_{\times}=h^{12}=h^{21}$ and $A_{\times}=A^{1}$ as well as $h_{+}=-h^{22}=h^{11}$ and $A_{+}=-A^{2}$. This is because the aforementioned equations can be elegantly cast as $[12,15]$ [30]

$\left(\square+\omega_{\mathrm{pl}}^{2} / c^{2}\right) A_{\lambda}=-B \partial_{\ell} h_{\lambda}, \quad \square h_{\lambda}=\kappa^{2} B \partial_{\ell} A_{\lambda}$,

where $\lambda \in\{+, \times\}, \ell$ is the third component, $\square=\partial_{t}^{2} /$ $c^{2}-\partial_{\ell}^{2}, \kappa=(16 \pi G)^{1 / 2} / c^{2}$. We include the plasma frequency $\omega_{\mathrm{pl}}=\sqrt{e^{2} n_{e} / m_{e}}$, which acts as an effective mass term and gives electromagnetic waves of frequency $\omega$ a refractive index $\mu=\sqrt{1-\omega_{\mathrm{pl}}^{2} / \omega^{2}}$ when $B \rightarrow 0$. Equation (1) also applies for arbitrary uniform fields with $B$ interpreted as the corresponding transverse component. See the Supplemental Material [31] for more details. Assuming a plane wave traveling in the positive direction with $\omega \geq \omega_{\mathrm{pl}}$, the exact solution of Eqs. (1) (see also Ref. [16]) can be written as

$$
\psi(t, \ell) \equiv\left(\begin{array}{c}
\sqrt{\mu} A_{\lambda} \\
\frac{1}{\kappa} h_{\lambda}
\end{array}\right)=e^{-i \omega t} e^{i K \ell} \psi(0,0),
$$

with $K$ being the Hermitian matrix

$$
K=\left(\begin{array}{cc}
\frac{\mu}{c} \sqrt{\omega^{2}+\left(\frac{\kappa B}{1+\mu}\right)^{2}} & -i \frac{\sqrt{\mu} \kappa B}{1+\mu} \\
i \frac{\sqrt{\mu} \kappa B}{1+\mu} & \frac{1}{c} \sqrt{\omega^{2}+\left(\frac{\kappa B}{1+\mu}\right)^{2}}
\end{array}\right) .
$$

It is convenient to introduce $\psi$ because its magnitude $|\psi(t, \ell)|^{2}$ is conserved. This easily follows from the unitarity of the matrix $\mathcal{U}(\ell)=e^{i K \ell}$. In particular, $\psi(0,0)=\left(0, h_{\lambda, 0} / \kappa\right)$ for a pure GW state entering the box, and, consequently, $\psi(t, \Delta \ell)=e^{-i \omega t}\left[\mathcal{U}_{12}(\Delta \ell)\right.$, $\left.\mathcal{U}_{22}(\Delta \ell)\right] h_{\lambda, 0} / \kappa$ after leaving the box. Since $\left|\mathcal{U}_{12}(\Delta \ell)\right|^{2}+$ $\left|\mathcal{U}_{22}(\Delta \ell)\right|^{2}=1$, the quantity $P(\Delta \ell) \equiv\left|\mathcal{U}_{12}(\Delta \ell)\right|^{2}$ can be interpreted as the probability of $\mathrm{GW}$ conversion after traversing a distance $\Delta \ell$. Simple algebra shows

$$
P(\Delta \ell)=\left|K_{12}\right|^{2} \ell_{\mathrm{osc}}^{2} \sin ^{2}\left(\Delta \ell / \ell_{\mathrm{osc}}\right),
$$

with $\ell_{\text {osc }}^{-1}=\sqrt{\omega^{2}(1-\mu)^{2} / c^{2}+\kappa^{2} B^{2}} / 2$. These expressions reduce to the approximated formulae previously found (see, e.g., Refs. [12,33]).

Although cosmic magnetic fields are not expected to be perfectly homogeneous, coherent oscillations take place in highly homogeneous patches, for which $\ell_{\text {osc }} \ll \Delta \ell$ and therefore $P(\Delta \ell)=\left|K_{12}\right|^{2} \ell_{\text {osc }}^{2} / 2$ on average. Taking into account inhomogeneities in $n_{e}$ [34] and $B$, the coherence of the $g \leftrightarrow \gamma$ oscillations is lost on distances larger than $\Delta \ell$, that is, the smallest distance on which $B$ and $n_{e}$ are uniform. Denoting the total distance traveled by the GW as $D$, this corresponds to traversing $N=D / \Delta \ell$ independent regions with a conversion probability $P(\Delta \ell)$ each. As long as $N P(\Delta \ell) \ll 1$, this gives a total conversion probability of $P(D)=D\left|K_{12}\right|^{2} \ell_{\text {osc }}^{2} /(2 \Delta \ell)[25,26]$, corresponding to an average conversion rate (i.e., probability per time) [31] given by

$$
\left\langle\Gamma_{g \leftrightarrow \gamma}\right\rangle=\frac{c\left|K_{12}\right|^{2} \ell_{\mathrm{osc}}^{2}}{2 \Delta \ell} .
$$

In the Supplemental Material [31] we demonstrate that this simple estimate correctly captures the essential features of a more involved computation based on the expected power spectrum of the magnetic field. Note that any additional inhomogeneities would further enhance the conversion rate by limiting the coherence of the $g \leftrightarrow \gamma$ oscillations.

We now include the effect of the Universe expansion during the dark ages. This is the period between photon decoupling and reionization, $z_{\mathrm{dec}} \simeq 1100 \gtrsim z \gtrsim z_{\text {rei }} \simeq 10$, beginning with the formation of the CMB and ending when the first stars were formed. During this time, the refractive index of $\mathrm{MHz}-\mathrm{GHz} \mathrm{CMB}$ photons is determined by the tiny electron density, with the contributions of neutral hydrogen, helium, and birefringence being subdominant [39-41]. This allows us to adopt Eq (5), after a few modifications. The conversion probability in an adiabatic expanding Universe is simply the line-of-sight integral of the rate

$$
\mathcal{P} \equiv \int_{\text {1.o.s. }}\left\langle\Gamma_{g \leftrightarrow \gamma}\right\rangle d t=\int_{0}^{z_{\text {ini }}} \frac{\left\langle\Gamma_{g \leftrightarrow \gamma}\right\rangle}{(1+z) H} d z
$$

where we use null geodesics $H d t=d T / T=d z /(1+z)$. Also, $z_{\mathrm{ini}} \leq z_{\mathrm{dec}}$ is an initial condition to be specified below and $H=H_{\mathrm{dec}}\left(T / T_{\mathrm{dec}}\right)^{3 / 2}$ is the Hubble parameter during the dark ages, which are matter dominated. Furthermore, the average magnetic energy density of the Universe $\rho_{B}=$ $B^{2} / 2$ redshifts as $\rho_{B}=\rho_{B 0}(1+z)^{4}$ [42]. Additionally, such a field is associated with a coherence length, $\lambda_{B}=\lambda_{B}^{0} /(1+z)$, because it is not expected to be homogeneous everywhere. Concerning these two quantities we emphasize three important facts here and refer the reader to Ref. [23] for a more comprehensive discussion: (i) a recent CMB analysis gives $B_{0} \lesssim 47 \mathrm{pG}$ [20], (ii) blazar 

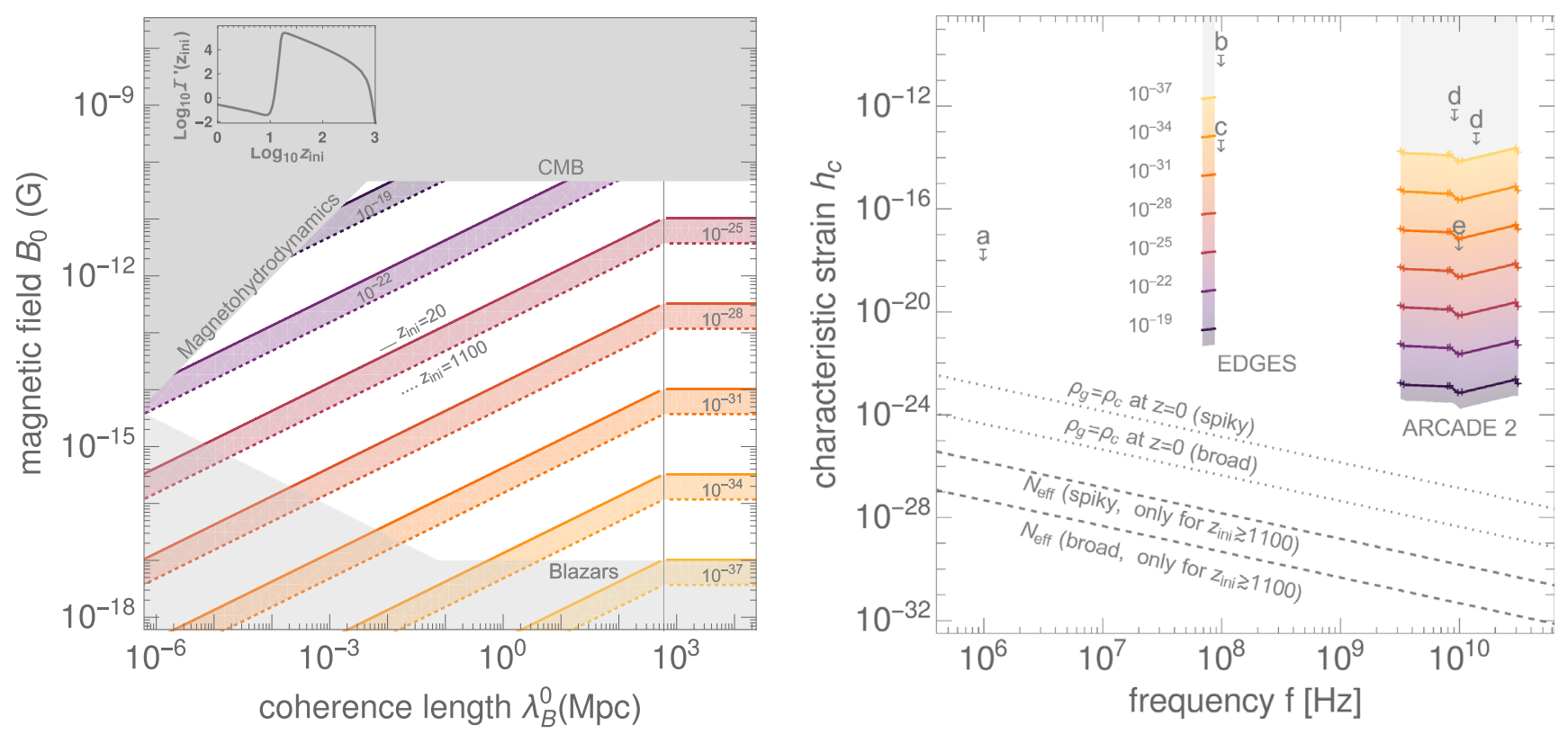

FIG. 2. Left: Parameter space for cosmic magnetic fields today. Gray shaded areas show the exclusion discussed in the text. The solid (dashed) colored curves indicate contour lines for the rescaled conversion probability, $\left(T_{0} / \omega_{0}\right)^{2} \mathcal{P}$. See Eqs. (6) and (7). Right: Upper bounds on the stochastic GW background derived from ARCADE2 and EDGES (this work), compared to existing laboratory bounds from (a) superconducting parametric converter [3], (b) waveguide [4], (c) $0.75 \mathrm{~m}$ interferometer [5], (d) magnon detector [6], and (e) magnetic conversion detector [7]. The solid lines indicate the allowed parameter space for cosmic magnetic fields, as given in the left panel. The dashed lines mark the $N_{\text {eff }}$ constraint for broad GW spectra and for a peaked spectrum with $\Delta \omega / \omega=10^{-3}$. For reference, the dotted lines indicate $\rho_{g}=\rho_{c}$.

observations strongly suggest a lower limit on $B_{0}$ [43] because otherwise their gamma-ray spectra cannot be explained under standard cosmological assumptions [1719,44], and (iii) magnetohydrodynamic turbulence damps out large magnetic fields at small distances, imposing an additional (theoretical) upper limit [23]. Figure 2 shows these constraints.

In addition, the electron number density during this epoch is $n_{e}(z)=n_{b 0}(1+z)^{3} X_{e}(z)$, where $n_{b 0}=0.251 \mathrm{~m}^{-3}$ is the baryon number density today [45] and $X_{e}(z)$ is the ionization fraction, taking values $1,0.68,0.0002$, and 0.15 at $z=0,10,20$, and 1100, respectively [46]. This gives plasma frequencies today, $\omega_{\mathrm{pl}, 0}$, lying in the $\mathrm{Hz}$ range, which allows us to take $1-\mu(z)=(1+z) X_{e}(z) \omega_{\mathrm{pl}, 0}^{2} /$ $\left(2 \omega_{0}^{2}\right) \ll 1$, for waves of frequency $\omega=\omega_{0}(1+z)$ with $\omega_{0} \sim \mathrm{GHz}$. Moreover, $B_{0} \lesssim 47 \mathrm{pG}$ results in the oscillation length being numerically dominated by the plasma frequency so that $\ell_{\mathrm{osc}}^{-1}=(1+z)^{2} X_{e}(z) \omega_{\mathrm{pl}, 0}^{2} /\left(4 \omega_{0} c\right)$. This gives $\ell_{\mathrm{osc}} \ll 1 \mathrm{pc} \ll \Delta \ell$, as anticipated above. Here, in order to account for electron inhomogeneities we conservatively take $\Delta \ell=\Delta \ell_{0} /(1+z)$ to be given by $\Delta \ell_{0}=\min \left[\lambda_{\mathrm{EQ}}, \lambda_{B}^{0}\right]$, where $\lambda_{\mathrm{EQ}} /(2 \pi)=95 \mathrm{Mpc}$ is the characteristic comoving scale for the onset of structure formation (corresponding to the perturbation mode entering the horizon at matter-radiation equality). Putting all this together, we obtain

$$
\mathcal{P} \simeq 6.3 \times 10^{-19}\left(\frac{B_{0}}{\mathrm{nG}}\right)^{2}\left(\frac{\omega_{0}}{T_{0}}\right)^{2}\left(\frac{\mathrm{Mpc}}{\Delta \ell_{0}}\right)\left(\frac{\mathcal{I}\left(z_{\text {ini }}\right)}{10^{6}}\right),
$$

with $T_{0} /(2 \pi)=2.725 \mathrm{~K} /(2 \pi)=56.78 \mathrm{GHz}$ and $\mathcal{I}\left(z_{\text {ini }}\right)=$ $\int_{0}^{z_{\text {ini }}} d z(1+z)^{-3 / 2} X_{e}^{-2}(z)$. The left panel of Fig. 2 displays contours of $\left(T_{0} / \omega_{0}\right)^{2} \mathcal{P}$ in the parameter space of cosmic magnetic fields. The inset shows $\mathcal{I}^{\prime}\left(z_{\text {ini }}\right)$, explaining the weak redshift dependence of $\mathcal{I}\left(z_{\text {ini }}\right)$, with the largest contribution arising from $z \sim 10$.

CMB distortions.-The CMB photon distribution, $f_{\gamma}(\omega, T)$, retains its equilibrium form during the dark ages, i.e., is given by a blackbody spectrum, $f_{\text {eq }}=1 /\left(e^{\omega / T}-1\right)$ with $\omega / T=\omega_{0} / T_{0}$. Our aim here is to calculate deviations from such a spectrum, $\delta f_{\gamma}=f_{\gamma}-f_{\text {eq }}$.

The spectrum of GWs is commonly characterized by $\Omega_{\mathrm{GW}}$, which parametrizes the corresponding energy density per logarithmic frequency bin. This quantity can be used to introduce - in an analogous manner to $f_{\gamma}$-the distribution function for GWs, $f_{g}$. More precisely, in terms of it, the energy density is given by

$\rho_{g}(T)=\int \frac{d \ln \omega}{\pi^{2}} \omega^{4} f_{g} \equiv \rho_{c}(T) \int d \ln \omega \Omega_{\mathrm{GW}}\left(\frac{\omega}{2 \pi}, T\right)$,

with $\rho_{c}(T)$ denoting the Universe total energy density. 
Both distributions satisfy the Boltzmann equation $\hat{L} f_{\gamma / g}= \pm\left\langle\Gamma_{g \leftrightarrow \gamma}\right\rangle\left(f_{g}-f_{\gamma}\right)$, where $\quad \hat{L} \equiv \partial_{t}-H \omega \partial_{\omega}=$ $-H\left(T \partial_{T}+\omega \partial_{\omega}\right)$ is the corresponding Lioville operator. Its solution leads to

$$
\delta f_{\gamma}\left(\omega_{0}, T_{0}\right)=\left[f_{g}\left(\omega_{\text {ini }}, T_{\text {ini }}\right)-f_{\text {eq }}\right] \mathcal{P}+\mathcal{O}\left(\mathcal{P}^{2}\right),
$$

with $\mathcal{P}$ defined as in Eq. (6). We solve the Boltzmann equations from an initial temperature $T=T_{\mathrm{ini}}$-when the photon distribution is a blackbody spectrum, i.e., $f_{\gamma}\left(\omega, T_{\text {ini }}\right)=f_{\text {eq }}\left(\omega / T_{\text {ini }}\right)$-until today. If decoupling is prior to the GW emission, the latter fixes $T_{\text {ini }}$. Otherwise, we set $T_{\mathrm{ini}}=T_{\mathrm{dec}}$ because the ionization fraction sharply drops after $z \sim z_{\text {dec }}$ rendering any prior contribution negligible. This is illustrated in the inset of Fig. 2 (left panel), which also shows that the conversion rate is anyways largely insensitive to the precise value of $T_{\text {ini }}$.

Equation (9) can alternatively be derived by considering the density-matrix formalism. In that case, $f_{\gamma}$ and $f_{g}$ are proportional to the diagonal entries of such a matrix, which evolves by means of the Hamiltonian associated with Eq. (3). See the Supplemental Material [31] for details. The fact that using both methods we obtain the same result-i.e., Eq. (9) - is reassuring and indicates that decoherence effects are properly taken into account [15]. Because of this as well as the way we treat inhomogeneities, our results differ from those of Ref. [26].

Constraints on the stochastic GW background.-In this Letter we focus on the Rayleigh-Jeans part of the CMB, i.e., $\omega \ll T$ implying $f_{\text {eq }} \simeq T / \omega$. In this regime, a subdominant GW contribution to the total radiation energy density is compatible with $f_{g} \gg f_{\gamma}$ [47], and can thus produce an enhancement of the low-frequency CMB tail through the first term of Eq. (9). More precisely, the assumption $f_{g}>f_{\gamma}$ translates to $\Omega_{\mathrm{GW}} / \Omega_{\gamma}>15 / \pi^{4}(\omega / T)^{3}$ as can be seen by rewriting Eq. (8) as $\Omega_{\mathrm{GW}}=\omega^{4} f_{g}(\omega, T) /$ $\left(\pi^{2} \rho_{c}\right)=\left(15 / \pi^{4}\right)(\omega / T)^{4} f_{g}(\omega, T) \Omega_{\gamma}$ with $\Omega_{\gamma}=\rho_{\gamma} / \rho_{c}=$ $\pi^{2} T^{4} /\left(15 \rho_{c}\right)$. Even a scale-invariant $\mathrm{GW}$ spectrum as small as $\Omega_{\mathrm{GW}} \simeq 10^{-15}$ implies $f_{g}>f_{\gamma}$ at, e.g., $\omega / T \simeq 10^{-3}$.

With $\omega \ll T$ and $f_{g} \gg f_{\gamma}$, Eq. (9) reads

$$
\frac{\delta f_{\gamma}}{f_{\gamma}}\left(\omega_{0}, T_{0}\right)=\frac{\pi^{4}}{15}\left(\frac{T}{\omega}\right)^{3} \mathcal{P} \frac{\Omega_{\mathrm{GW}}}{\Omega_{\gamma}} .
$$

For a given detector sensitivity $\delta f_{\gamma} / f_{\gamma}$ and a given value of the conversion probability $\mathcal{P}$, relation (10) sets stringent bounds on the GW spectrum, which can be expressed in terms of the characteristic strain by means of [49]

$$
h_{c}=\left(\frac{3 H_{0}^{2}}{4 \pi^{2}} \Omega_{\mathrm{GW}} f^{-2}\right)^{1 / 2} \text {. }
$$

This is related to the one-sided power spectral density $S_{h}$ as $h_{c}=\sqrt{f S_{h}(f)}$. Figure 2 contrasts the resulting constraints with existing bounds in the literature.
$N_{\text {eff }}$ bound.-GWs contribute to the energy budget of the Universe in the form of radiation and are as such constrained by the BBN and $\mathrm{CMB}$ bounds on the effective number of massless degrees of freedom $N_{\text {eff }}$ [50],

$$
\rho_{g}(T) \leq \frac{7}{8}\left(\frac{4}{11}\right)^{4 / 3} \Delta N_{\mathrm{eff}} \rho_{\gamma}(T)
$$

with $\Delta N_{\text {eff }} \lesssim 0.1[45,51]$. For a spectrum $\Omega_{\mathrm{GW}}$ which is approximately scale invariant between $f_{\min }$ and $f_{\max }$ with $\ln \left(f_{\max } / f_{\min }\right) \sim \mathcal{O}(1)$, this implies

$$
\frac{\Omega_{\mathrm{GW}}}{\Omega_{\gamma}} \lesssim \frac{7}{8}\left(\frac{4}{11}\right)^{4 / 3} \Delta N_{\mathrm{eff}},
$$

whereas for a narrow spectrum peaked at $\tilde{\omega}$ with width $\Delta \tilde{\omega} \lesssim \tilde{\omega}$ this bound is relaxed by a factor $(\tilde{\omega} / \Delta \tilde{\omega})$. Note that this bound applies only to GWs present already at CMB decoupling.

Probing the Rayleigh-Jeans tail of the CMB spectrum.Below $\omega_{0} / T_{0} \sim 10^{-2}$, galactic foregrounds dominate the radio sky. Here we focus on the results reported by ARCADE2 [28] which covers the sweet spot of the lowfrequency Rayleigh-Jeans spectrum before galactic foregrounds become important $\left[f=\omega_{0} /(2 \pi)=3,8,10,30\right.$, and $90 \mathrm{GHz}$ ] and by EDGES [29], which is a recent measurement of the global $21 \mathrm{~cm}$ absorption signal at $78 \mathrm{MHz}$.

ARCADE2 was a balloon experiment equipped with a radio receiver measuring the blackbody temperature of sky [28]. The cleanest frequency band is around $10 \mathrm{GHz}$ enabling a $\mathrm{mK}$ resolution, $\delta f_{\gamma} / f_{\gamma}=\delta T / T_{\mathrm{CMB}} \lesssim 4 \times 10^{-4}$ at $\omega_{0} / T_{0} \simeq 0.18$. At smaller frequencies, ARCADE2 observed a significant radio excess beyond the expected galactic foreground whose origin remains an open question (see, e.g., Refs. [52,53]). Assuming that this excess is entirely astrophysical, we can impose an upper bound on an additional contribution from a stochastic GW background using the 3, 8, 10, and $30 \mathrm{GHz}$ frequency bands. In Fig. 2, these frequencies are marked by crosses, the solid lines connecting them serve only to guide the eye.

Recently, the first observation of the global (i.e., skyaveraged) $21 \mathrm{~cm}$ absorption signal was reported by the EDGES Collaboration [29]. The absorption feature was found to be roughly twice as strong as previously expected, which if true, would indicate that either the primordial gas was significantly colder or the radiation background was significantly hotter than expected. Conservatively, we may assume that the deviation from the expected value is due to foreground contamination, and place a bound on any stochastic GW background by using $\delta f_{\gamma} / f_{\gamma} \lesssim 1$ at $\omega / T=$ $1.4 \times 10^{-3}(78 \mathrm{MHz})$. The width of the observed absorption feature $(19 \mathrm{MHz})$ determines the width of the frequency coverage. 
Discussion.-Cosmological sources of GWs typically produce stochastic GW backgrounds with a frequency roughly related to the comoving Hubble horizon at the time of production. Processes in the very early Universe at energy scales far beyond the reach of colliders thus generically produce $\mathrm{GWs}$ in the $\mathrm{MHz}$ and $\mathrm{GHz}$ regime. Despite the large amount of redshift, these violent processes can produce sizable GW signals, saturating the $N_{\text {eff }}$ bound (12). Some examples are axion inflation [54,55], metastable cosmic strings [57] and evaporating light primordial black holes $[58,59]$. Further significant contributions may be expected from preheating [60-64] and first order phase transitions occurring above $10^{7} \mathrm{GeV}$ [65-69]. The sensitivity of radio telescopes can, however, not yet compete with the cosmological $N_{\text {eff }}$ bound, unless one considers essentially monochromatic signals (which may arise, e.g., from large monochromatic scalar perturbations $[70,71])$.

Since the dominant contribution to $\mathcal{P}$ arises around reionization, particularly interesting targets are GW sources active around $10 \lesssim z \lesssim 10^{3}$, which would not be constrained by the $N_{\text {eff }}$ bound. During the dark ages, there is no generic reason to expect $\mathrm{GW}$ production in the $\mathrm{GHz}$ regime but there are models which predict such a signal for suitable parameter choices. For example, mergers of light primordial black holes in this epoch (with masses of about $10^{-9 . .-7} M_{\odot}$ ) would result in $\mathrm{GHz} \mathrm{GW}$ signals today [49], see Refs. [72,73] for a discussion of possible rates. Superradiant axion clouds around spinning black holes yield an essentially monochromatic GW signal with $f \lesssim$ $\mathrm{MHz}$ [74-76], with higher frequency possible when considering primordial black holes with masses below the Chandrasekhar limit.

We emphasize that the use of radio telescopes allows us to search for GWs in a wide frequency regime. While the absence of any excess radiation can already constrain some models under the assumption of strong cosmic magnetic fields, the potential of this method will truly unfold with further improvements in the sensitivity of radio telescopesdriven in particular by the advances in $21 \mathrm{~cm}$ cosmologyor in the case of a positive detection of excess radiation.

An example of future advances in radio astronomy is the case of the Square Kilometer Array (SKA). Assuming an effective area per antenna temperature of at least $10^{2} \mathrm{~m}^{2} / \mathrm{K}$ $[77,78]$ in the $0.1-10 \mathrm{GHz}$ range, a few hours of observation will lead to sensitivities in the ballpark of $\mu \mathrm{Jy}$, which must be compared against $\mathrm{CMB}$ fluxes of at least $10^{3} \mathrm{Jy}$. SKA measurements are thus very promising although sufficient foreground subtraction will be extremely challenging.

It is a pleasure to thank Nancy Aggarwal, Sebastien Clesse, Mike Cruise, Hartmut Grote, and Francesco Muia for insightful discussions on the Gertsenshtein effect and high-frequency GW sources at the ICTP workshop
"Challenges and opportunities of high-frequency gravitational wave detection." Likewise, we would also like to thank Torsten Bringmann, Damian Ejlli, Kohei Kamada, and Kai Schmidt-Hoberg. This work was partially funded by the Deutsche Forschungsgemeinschaft under Germany's Excellence Strategy-EXC 2121 "Quantum Universe"390833306. C. G. C. is supported by the Alexander von Humboldt Foundation.

*valerie.domcke@cern.ch †camilo.garcia.cely@desy.de

[1] M. Maggiore, Gravitational Waves. Vol. 2: Astrophysics and Cosmology (Oxford University Press, Oxford, 2018).

[2] C. Caprini and D. G. Figueroa, Cosmological backgrounds of gravitational waves, Classical Quantum Gravity 35, 163001 (2018).

[3] C. Reece, P. Reiner, and A. Melissinos, Observation of 4 10-17 cm harmonic displacement using a $10 \mathrm{GHz}$ superconducting parametric converter, Phys. Lett. 104A, 341 (1984).

[4] A. Cruise and R. Ingley, A prototype gravitational wave detector for 100-MHz, Classical Quantum Gravity 23, 6185 (2006).

[5] T. Akutsu et al., Search for a Stochastic Background of 100-MHz Gravitational Waves with Laser Interferometers, Phys. Rev. Lett. 101, 101101 (2008).

[6] A. Ito and J. Soda, A formalism for magnon gravitational wave detectors, Eur. Phys. J. C 80, 545 (2020).

[7] A. M. Cruise, The potential for very high-frequency gravitational wave detection, Classical Quantum Gravity 29, 095003 (2012).

[8] A. Ejlli, D. Ejlli, A. M. Cruise, G. Pisano, and H. Grote, Upper limits on the amplitude of ultra-high-frequency gravitational waves from graviton to photon conversion, Eur. Phys. J. C 79, 1032 (2019).

[9] M. E. Gertsenshtein, Wave resonance of light and gravitational waves, Sov. Phys. JETP 14, 84 (1962), http://www .jetp.ac.ru/cgi-bin/e/index/e/14/1/p84?a=list.

[10] D. Boccaletti, V. De Sabbata, P. Fortini, and C. Gualdi, Conversion of photons into gravitons and vice versa in a static electromagnetic field, Il Nuovo Cimento B 70, 129 (1970), https://www.osti.gov/biblio/4070463.

[11] W. K. De Logi and A. R. Mickelson, Electrogravitational conversion cross-sections in static electromagnetic fields, Phys. Rev. D 16, 2915 (1977).

[12] G. Raffelt and L. Stodolsky, Mixing of the photon with low mass particles, Phys. Rev. D 37, 1237 (1988).

[13] P. Macedo and A. Nelson, Propagation of gravitational waves in a magnetized plasma, Phys. Rev. D 28, 2382 (1983).

[14] D. Fargion, Prompt and delayed radio bangs at kilohertz by SN1987A: A test for gravitation-photon conversion, Gravitation Cosmol. 1, 301 (1995), https://arxiv.org/abs/ astro-ph/9604047.

[15] A. D. Dolgov and D. Ejlli, Conversion of relic gravitational waves into photons in cosmological magnetic fields, J. Cosmol. Astropart. Phys. 12 (2012) 003. 
[16] D. Ejlli, Graviton-photon mixing. Exact solution in a constant magnetic field, J. High Energy Phys. 06 (2020) 029.

[17] A. Neronov and I. Vovk, Evidence for strong extragalactic magnetic fields from Fermi observations of $\mathrm{TeV}$ blazars, Science 328, 73 (2010).

[18] F. Tavecchio, G. Ghisellini, L. Foschini, G. Bonnoli, G. Ghirlanda, and P. Coppi, The intergalactic magnetic field constrained by Fermi/LAT observations of the TeV blazar 1ES 0229 + 200, Mon. Not. R. Astron. Soc. 406, L70 (2010).

[19] K. Takahashi, M. Mori, K. Ichiki, S. Inoue, and H. Takami, Lower bounds on magnetic fields in intergalactic voids from long-term GeV-TeV light curves of the Blazar Mrk 421, Astrophys. J. 771, L42 (2013).

[20] K. Jedamzik and A. Saveliev, Stringent Limit on Primordial Magnetic Fields from the Cosmic Microwave Background Radiation, Phys. Rev. Lett. 123, 021301 (2019).

[21] Planck Collaboration, Planck 2015 results. XIX. Constraints on primordial magnetic fields, Astron. Astrophys. 594, A19 (2016).

[22] M. S. Pshirkov, P. G. Tinyakov, and F. R. Urban, New Limits on Extragalactic Magnetic Fields from Rotation Measures, Phys. Rev. Lett. 116, 191302 (2016).

[23] R. Durrer and A. Neronov, Cosmological magnetic fields: Their generation, evolution and observation, Astron. Astrophys. Rev. 21, 62 (2013).

[24] P. Chen, Resonant Photon-Graviton Conversion and Cosmic Microwave Background Fluctuations, Phys. Rev. Lett. 74, 634 (1995).

[25] A. N. Cillis and D. D. Harari, Photon-graviton conversion in a primordial magnetic field and the cosmic microwave background, Phys. Rev. D 54, 4757 (1996).

[26] M. S. Pshirkov and D. Baskaran, Limits on high-frequency gravitational wave background from its interplay with large scale magnetic fields, Phys. Rev. D 80, 042002 (2009).

[27] T. Fujita, K. Kamada, and Y. Nakai, Gravitational waves from primordial magnetic fields via photon-graviton conversion, Phys. Rev. D 102, 103501 (2020).

[28] D. J. Fixsen, A. Kogut, S. Levin, M. Limon, P. Lubin, P. Mirel et al., Arcade 2 Measurement of the Absolute Sky Brightness at 3-90 GHz, Astrophys. J. 734, 5 (2011).

[29] J. D. Bowman, A. E. E. Rogers, R. A. Monsalve, T. J. Mozdzen, and N. Mahesh, An absorption profile centred at 78 megahertz in the sky-averaged spectrum, Nature (London) 555, 67 (2018).

[30] The first relation in Eqs. (1) differs from that of Ref. [27] in the negative sign.

[31] See Supplemental Material at http://link.aps.org/ supplemental/10.1103/PhysRevLett.126.021104 for the discussion showing that the effect of the magnetic field on the wave velocity is negligible, which includes a reference to Ref. [32].

[32] LIGO Scientific, Virgo, Fermi-GBM, and INTEGRAL Collaboration, Gravitational waves and gamma-rays from a binary neutron star merger: GW170817 and GRB 170817A, Astrophys. J. 848, L13 (2017).

[33] D. Ejlli and V. R. Thandlam, Graviton-photon mixing, Phys. Rev. D 99, 044022 (2019).

[34] See the discussion in the Supplemental Material [31], which includes Refs. [35-38].
[35] B. Venhlovska and B. Novosyadlyj, Power spectrum of electron number density perturbations at cosmological recombination epoch, J. Phys. Stud. 12, 3901 (2008).

[36] C. Dvorkin, K. Blum, and M. Zaldarriaga, Perturbed recombination from dark matter annihilation, Phys. Rev. D 87, 103522 (2013).

[37] D. P. Finkbeiner, S. Galli, T. Lin, and T. R. Slatyer, Searching for dark matter in the CMB: A compact parametrization of energy injection from new physics, Phys. Rev. D 85, 043522 (2012).

[38] E. D. Carlson and W. Garretson, Photon to pseudoscalar conversion in the interstellar medium, Phys. Lett. B 336, 431 (1994).

[39] P. Chen and T. Suyama, Constraining primordial magnetic fields by CMB photon-graviton conversion, Phys. Rev. D 88, 123521 (2013).

[40] K. E. Kunze and M. A. Vázquez-Mozo, Constraints on hidden photons from current and future observations of CMB spectral distortions, J. Cosmol. Astropart. Phys. 12 (2015) 028.

[41] A. Mirizzi, J. Redondo, and G. Sigl, Microwave Background constraints on mixing of photons with hidden photons, J. Cosmol. Astropart. Phys. 03 (2009) 026.

[42] In Eq. (5), $B^{2} \rightarrow 2 B^{2} / 3$ extracts the transverse component.

[43] C. Caprini and S. Gabici, Gamma-ray observations of blazars and the intergalactic magnetic field spectrum, Phys. Rev. D 91, 123514 (2015).

[44] W. Chen, J. H. Buckley, and F. Ferrer, Search for GeV -Ray Pair Halos Around Low Redshift Blazars, Phys. Rev. Lett. 115, 211103 (2015).

[45] Planck Collaboration, Planck 2018 results. VI. Cosmological parameters, Astron. Astrophys. 641, A6 (2020).

[46] For numerical computations, we use the values reported in Ref. [40].

[47] See Ref. [48] for a related argument for decaying dark matter.

[48] M. Pospelov, J. Pradler, J. T. Ruderman, and A. Urbano, Room for New Physics in the Rayleigh-Jeans Tail of the Cosmic Microwave Background, Phys. Rev. Lett. 121, 031103 (2018).

[49] M. Maggiore, Gravitational Waves. Vol. 1: Theory and Experiments, Oxford Master Series in Physics (Oxford University Press, Oxford, 2007).

[50] L. Pagano, L. Salvati, and A. Melchiorri, New constraints on primordial gravitational waves from Planck 2015, Phys. Lett. B 760, 823 (2016).

[51] R. H. Cyburt, B. D. Fields, K. A. Olive, and T.-H. Yeh, Big bang nucleosynthesis: 2015, Rev. Mod. Phys. 88, 015004 (2016).

[52] M. Seiffert, D. J. Fixsen, A. Kogut, S. M. Levin, M. Limon, P. M. Lubin et al., Interpretation of the Arcade 2 absolute sky brightness measurement, Astrophys. J. 734, 6 (2011).

[53] C. Feng and G. Holder, Enhanced global signal of neutral hydrogen due to excess radiation at cosmic dawn, Astrophys. J. 858, L17 (2018).

[54] N. Barnaby and M. Peloso, Large Nongaussianity in Axion Inflation, Phys. Rev. Lett. 106, 181301 (2011).

[55] The dominant GW signal of axion inflation may also be related to its subsequent preheating phase [56], also leading to $\mathrm{MHz}$ to $\mathrm{GHz}$ signal which can easily saturate the $N_{\text {eff }}$ bound. 
[56] P. Adshead, J. T. Giblin, M. Pieroni, and Z. J. Weiner, Constraining axion inflation with gravitational waves from preheating, Phys. Rev. D 101, 083534 (2020).

[57] W. Buchmuller, V. Domcke, H. Murayama, and K. Schmitz, Probing the scale of grand unification with gravitational waves, Phys. Lett. B 809, 135764 (2020).

[58] R. Anantua, R. Easther, and J. T. Giblin, GUT-Scale Primordial Black Holes: Consequences and Constraints, Phys. Rev. Lett. 103, 111303 (2009).

[59] R. Dong, W. H. Kinney, and D. Stojkovic, Gravitational wave production by Hawking radiation from rotating primordial black holes, J. Cosmol. Astropart. Phys. 10 (2016) 034.

[60] A. Dolgov and D. Kirilova, On particle creation by a time dependent scalar field, Sov. J. Nucl. Phys. 51, 172 (1990), https://lib-extopc.kek.jp/preprints/PDF/1989/8908/8908442 .pdf.

[61] J. H. Traschen and R. H. Brandenberger, Particle production during out-of-equilibrium phase transitions, Phys. Rev. D 42, 2491 (1990).

[62] L. Kofman, A. Linde, and A. A. Starobinsky, Reheating after Inflation, Phys. Rev. Lett. 73, 3195 (1994).

[63] L. Kofman, A. D. Linde, and A. A. Starobinsky, Towards the theory of reheating after inflation, Phys. Rev. D 56, 3258 (1997).

[64] D. G. Figueroa and F. Torrenti, Gravitational wave production from preheating: parameter dependence, J. Cosmol. Astropart. Phys. 10 (2017) 057.

[65] E. Witten, Cosmic separation of phases, Phys. Rev. D 30, 272 (1984).

[66] C. J. Hogan, Gravitational radiation from cosmological phase transitions, Mon. Not. R. Astron. Soc. 218, 629 (1986).

[67] M. Punturo et al., The Einstein telescope: A third-generation gravitational wave observatory, Classical Quantum Gravity 27, 194002 (2010).
[68] S. Hild et al., Sensitivity studies for third-generation gravitational wave observatories, Classical Quantum Gravity 28, 094013 (2011).

[69] LIGO Scientific Collaboration, Exploring the sensitivity of next generation gravitational wave detectors, Classical Quantum Gravity 34, 044001 (2017).

[70] E. Bugaev and P. Klimai, Induced gravitational wave background and primordial black holes, Phys. Rev. D 81, 023517 (2010).

[71] R. Saito and J. Yokoyama, Gravitational-wave constraints on the abundance of primordial black holes, Prog. Theor. Phys. 123, 867 (2010).

[72] M. Raidal, C. Spethmann, V. Vaskonen, and H. Veerme, Formation and evolution of primordial black hole binaries in the early universe, J. Cosmol. Astropart. Phys. 02 (2019) 018.

[73] A. D. Gow, C. T. Byrnes, A. Hall, and J. A. Peacock, Primordial black hole merger rates: Distributions for multiple ligo observables, J. Cosmol. Astropart. Phys. 01 (2020) 031.

[74] A. Arvanitaki, S. Dimopoulos, S. Dubovsky, N. Kaloper, and J. March-Russell, String axiverse, Phys. Rev. D 81, 123530 (2010).

[75] A. Arvanitaki and S. Dubovsky, Exploring the string axiverse with precision black hole physics, Phys. Rev. D 83, 044026 (2011).

[76] A. Arvanitaki and A. A. Geraci, Detecting High-Frequency Gravitational Waves with Optically Levitated Sensors, Phys. Rev. Lett. 110, 071105 (2013).

[77] R. Braun, A. Bonaldi, T. Bourke, E. Keane, and J. Wagg, Anticipated performance of the square kilometre arrayPhase 1 (SKA1), arXiv:1912.12699.

[78] See also https://www.skatelescope.org/wp-content/ uploads/2014/03/SKA-TEL_SCI-SKO-SRQ-001-1_Level_0_ Requirements-1.pdf. 